\title{
Development and validation of an instrument assessing Health System Responsiveness of family planning services in Sri Lanka
}

\author{
W L S P Perera ${ }^{1}$, Rohini Seneviratne ${ }^{2}$, Thushara Fernando ${ }^{3}$
}

'Acting National Coordinator, Disaster Preparedness and Response Division, Ministry of Health, Sri Lanka.

${ }^{2} \mathrm{Head}$, Department of Community Medicine, Faculty of Medicine, University of Colombo, Sri Lanka. ${ }^{3}$ Medical Officer, Human Resource for Health, South East Asia Regional Office, World Health Organization, New Delhi, India.

\section{Abstract}

Health System Responsiveness (HSR) has been described by the World Health Organization (WHO) as how health systems meet non-medical expectations of its service seekers. It is a multi-domain concept where assessment of responsiveness is made by service seekers. The objective of this study was to develop and validate a Health System Responsiveness Assessment Questionnaire (HESRAQ) in relation to family planning (FP) services in Sri Lanka. Both qualitative and quantitative methodologies were used in the FP delivery settings. Focus group discussions (FGD), in-depth interviews and a modified Delphi method were used. Cross sectional surveys were conducted to carryout exploratory and confirmatory factor analysis (EFA and CFA). Literature reviews, eight FGDs and 45 in-depth interviews were conducted where 15 domains were generated. Eight domains were finalized using a modified Delphi method. A total of ninety two items were generated in the finalized domains. However, 28 items were finalized with expert opinion and EFA with the ratings of 255 respondents. The 28 item instrument was validated with CFA with the rating of 200 respondents, with the six domain model being the most valid $(\chi 2=1272, \mathrm{df}=335, \mathrm{p}<0.000, \mathrm{GFI}=0.69)$ The HESRAQ also showed a high level of reliability for all domains (Cronbach's alpha $>0.877$ ). The HESRAQ is a valid and reliable tool to assess HSR of family planning services in Sri Lanka.

Keywords: Health system responsiveness, Non-medical expectations, Family planning services

\section{Introduction}

Health systems play a pivotal role in peoples' lives. People have both 'medical' and 'non-medical' expectations from the health system. While the primary intention of a health system is to provide services of the highest medical competence, non-medical aspects are often ignored. ${ }^{1}$

Today, health systems have come under criticism for not meeting peoples' expectations on non-medical aspects, despite providing the best care in the technical sense. 'Time has come for health systems to pay more attention to the non-medical expectations of their service seekers. In this background, the World health Organization (WHO) introduced the concept of Health System Responsiveness (HSR) which was defined in the World Health Report 2000 as how health systems respond to legitimate non-medical expectations of its service seekers. ${ }^{1}$

The WHO described HSR as a multi-domain concept and identified 'prompt attention', 'dignity', 'communication', 'confidentiality', 'choice of the provider', 'social support',

\section{Practice points}

- HRS is a novel and an important concept in assessing performance of health systems

- As a validated instrument was unavailable, it is therefore important to develop a valid instrument

- Using both qualitative and quantitative methodologies, the HESRAQ was developed and validated

- The HESRAQ is a valid and reliable tool to assess the HSR of FP services in Sri Lanka

- The tool can be used to evaluate the expectations of service seekers of other healthcare programs

'autonomy' and 'basic amenities' as component domains. ${ }^{1} \mathrm{~A}$ module, developed on a global perspective, was used to assess HSR and responsiveness of member states, was assessed by the WHO in 2002. ${ }^{1}$ However, this module developed on a global perspective does not adequately

Corresponding: Dr. W L S P Perera, Acting National Coordinator, Disaster Preparedness and Response Division, Ministry of Health, Sri Lanka. E-mail: sugandhika_perera@yahoo.co.in; sugandhikaperera@gmail.com. 
assess the HSR of the health system in Sri Lanka. No other instrument has been developed to assess HSR; consequently, responsiveness has not been scientifically assessed after 2002. This study was designed with the objective of developing an instrument to assess HSR in the Sri Lankan context. The study was confined to a single service, particularly family planning (FP) service, which was selected for its sensitive nature where 'non-medical' expectations of clients are as high as 'medical' expectations. The government family planning service, being the largest provider of FP services in Sri Lanka, was selected for the study.

\section{Methods and Materials}

Study setting

Gampaha district was selected randomly from 26 districts.

\section{Identification of domains}

Initially, a literature review was conducted to identify the domains of HSR leading to the selection of eight domains. The domain list was expanded with focus group discussions (FGD) ranging from nine to twelve FP clients in each group. Further, in-depth interviews were carried out with stakeholders including clients, eligible couples with unmet needs in FP, service providers, medical administrators, community physicians and representatives of civil societies. Purposive sampling was adopted to select respondents. A total of eight FGDs and 45 interviews were conducted before the theoretical saturation point was reached (i.e. where no new data was generated). Notes were taken during the FGDs and interviews, and transcripts were made and coded to identify the main themes.

Domains were finalized with a modified Delphi method, by post, and the views of 40 participants were obtained in two iterations of independent rating of domains. Participants comprised respondents from categories which participated in FGDs and interviews. Respondents rated the importance of domains on a five-point scale ranging from 'very important' to 'least important'. A median score of 3 (important) was considered the cutoff for inclusion in the next step.

\section{Identification of the items}

Following finalization of domains, items were identified under those domains. Items were generated with a review of literature, FGDs and in-depth interviews. Subsequently, items were reduced with the opinion of ten experts in HSR, FP service provision, medical administration and policy planning. Experts rated the importance of items on a fivepoint scale ranging from 'very important' to 'least important'. Items obtaining a median score of 3 (important) were included in the exploratory factor analysis (EFA) for further reduction. Clients seeking FP services from clinics were invited to rate items, selected by the experts, on a similar scale for the EFA. The number of respondents was five times the number of items. ${ }^{2}$ Respondents were clients attending all FP clinics in the study setting and EFA was conducted with SPSS 15.0.
Validation of HESRAQ

Judgmental validity of HESRAQ was assessed during the development process since face, content and consensual validity was incorporated in the development methods.

\section{Construct validity}

Confirmatory factor analysis (CFA) was applied to assess the construct validity of the instrument. ${ }^{3}$ To carryout CFA, clients seeking services were invited to rate the responsiveness of clinics on a five-point scale ranging from 'very good' to 'very bad'. The number of clients was over five times the number of items $(n=200){ }^{3}$ LISREL 8.8 software was used in the analysis.

\section{Results and Analysis}

Identification of domains

Eight domains were generated in the literature review, four in the FGDs and three during the interviews. The eight domains described in literature were included and nomenclature of five was changed to suit the local context. 'Prompt attention', 'dignity', 'social support', 'autonomy' and 'basic amenities' were renamed as 'ease of access', 'treated with dignity', 'family involvement', 'choice of a method' and 'clinic environment' respectively. During FGDs, the domains of 'personality of provider', 'personality of client', 'courteousness of provider' and 'leadership/administrative qualities of Medical Officer in Charge (MOIC) of the clinic' were added. The domains added during the interviews were: 'perceived adequacy of staff', 'perceived adequacy of drugs/equipment' and 'adoption of productivity concept'.

A total of fifteen domains were identified; nine during Delphi process: 'ease of access', 'treated with dignity', 'choice of method', 'clinic environment', 'family involvement, choice of provider', 'communication', 'confidentiality and administrative qualities of the MOIC'. Selected domains in the first iteration were sent for reevaluation; however, 'administrative qualities of MOIC' failed to obtain the median of 3 . Other eight domains were finalized as components of HSR. Response rates were 53\% $(n=23)$ and $45 \%(n=18)$ in the respective rounds.

\section{Identification of items}

A total of 92 items were generated under the eight domains following literature review, FGDs and interviews. Fifty two items were generated in the literature review, 21 during the FGDs and 19 items from the interviews. During item reduction, 51 items were selected by experts. EFA was conducted on the rating of the 51 items by a sample of 255 clients during the cross sectional survey. All participants of the cross sectional surveys were married females who sought services from FP clinics.

During EFA, the number of factors to be extracted was specified as eight, since eight domains were identified earlier. The Bartlett's test for sphericity significantly confirmed the factorability of data $(\chi 2=3445.737, \mathrm{df}=378$, 
$\mathrm{p}<0.001)^{2}$ A Keiser-Mayer-Olkin measure of above 0.7 indicates the suitability of data for EFA and the measure was 0.763 in the present study. Varimax rotation was used and a coefficient value of over 0.5 was considered in deciding the cutoff, since values above 0.3 is acceptable. ${ }^{2}$

Eight factors were named as domains based on the item loading. Five out of eight items developed under the 'treated with dignity' loaded into factor one. All six items of the 'ease of access' domain were loaded into factor two. Four out of eight items generated under the 'clinic environment' domain were loaded into factor thee. Five out of ten generated items of 'communication' were loaded into factor four. Two of the four items of 'confidentiality' were loaded to the fifth factor and three of the four items developed under 'choice of the provider' were loaded into factor six. Two of the five items under 'choice of the method' were loaded into the seventh factor and the last factor was considered as 'family involvement ${ }^{\prime}$ and one item generated under this domain was loaded into the eighth factor. Twenty eight items were loaded into eight factors logically and it was decided to retain an order that all items could fit logically. Detailed distribution of the items is shown in Table 1. The instrument consisting of 28 items was then validated for Sri Lanka.

Table 1: Item distribution

\begin{tabular}{|c|c|c|c|c|c|c|c|c|}
\hline Items & 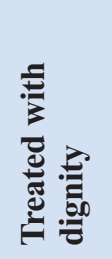 & 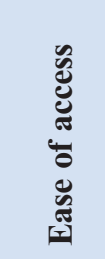 & 竞 & 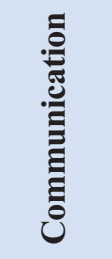 & 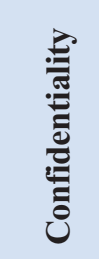 & 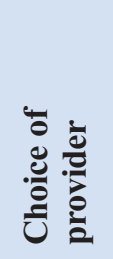 & 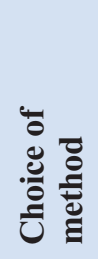 & 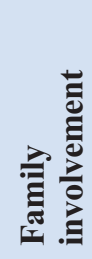 \\
\hline Treated with dignity by service provider & .671 & -.135 & -.179 & .199 & -.037 & -.061 & .101 & .223 \\
\hline Treated with dignity by other staff & .518 & -.073 & -.141 & .464 & -.026 & -.029 & -.058 & .297 \\
\hline Treated kindly & .610 & -.098 & -.020 & .465 & -.034 & .009 & -.041 & -.175 \\
\hline Listened to & .644 & -.064 & .072 & .238 & -.120 & .127 & -.162 & -.386 \\
\hline Privacy during physical examinations & .792 & -.088 & -.141 & .106 & -.005 & -.072 & -.139 & .153 \\
\hline Treated friendly & .409 & -.130 & -.177 & .634 & .003 & -.142 & .068 & .289 \\
\hline Greeted by staff & -.177 & 0.063 & .175 & .864 & .149 & .067 & .054 & .052 \\
\hline Treated with courtesy & -.314 & -.208 & .275 & .761 & .150 & .180 & .088 & .111 \\
\hline Cultural values respected & -.042 & .143 & .151 & -.104 & .942 & .043 & .047 & -.046 \\
\hline Total time spent to obtain services & .290 & .586 & -.069 & .360 & -.079 & -.158 & -.082 & -.107 \\
\hline Travel time & .105 & .924 & -.008 & -.005 & .040 & -.123 & .075 & .055 \\
\hline Convenience of time & -.102 & .836 & .148 & .169 & .111 & .072 & .128 & -.022 \\
\hline Convenience of day & -.380 & .741 & .320 & -.199 & .143 & .246 & .053 & .076 \\
\hline Time waited till registered & -.116 & .806 & .206 & -.052 & .046 & .087 & -.031 & -.197 \\
\hline Total time waiting & -.321 & .760 & .272 & -.205 & .106 & .217 & .067 & .194 \\
\hline Facilities for examination & -.336 & .232 & .843 & -.128 & .096 & .038 & .114 & .113 \\
\hline Toilet facilities & .029 & .110 & .594 & -.064 & .127 & .298 & .152 & -.171 \\
\hline General cleanliness & -.140 & .179 & .889 & -.034 & .064 & -.011 & .101 & -.004 \\
\hline Seating facilities & -.185 & .220 & .894 & -.121 & .114 & .047 & .075 & .040 \\
\hline Acceptability of buildings & -.211 & .824 & .211 & .138 & .077 & .178 & .054 & .025 \\
\hline Cleanliness of linen & .714 & -.132 & -.253 & .048 & -.148 & -.141 & .194 & .159 \\
\hline Space availability & .734 & -.048 & .017 & .171 & -.072 & .137 & -.094 & -.068 \\
\hline Windows for ventilation & .757 & -.099 & -.121 & .280 & -.015 & -.116 & -.123 & .128 \\
\hline Answering problems & .344 & -.185 & -.188 & .599 & -.075 & -.168 & -.088 & -.010 \\
\hline
\end{tabular}




\begin{tabular}{|c|c|c|c|c|c|c|c|c|}
\hline Able to obtain information .408 & .408 & -.135 & -.033 & .556 & -.161 & .121 & -.067 & -.049 \\
\hline Information on current FP method & .416 & -.066 & -.021 & .578 & -.089 & .068 & .141 & -.191 \\
\hline Information on side effects & .184 & .080 & -.067 & .591 & .158 & .373 & -.191 & -.040 \\
\hline Time for questions & .305 & .478 & .060 & .550 & -.027 & -.068 & -.092 & -.044 \\
\hline Information on all methods & .832 & -.182 & .206 & -.051 & .063 & .140 & .147 & .100 \\
\hline Time to clarify information & .756 & -.117 & -.201 & .009 & .003 & -.110 & -.023 & .086 \\
\hline Consent obtained & .545 & -.069 & -.002 & .297 & .004 & .175 & -.076 & -.199 \\
\hline Encouraged questioning & .111 & -.081 & -.045 & .126 & -.066 & .054 & .725 & -.256 \\
\hline Not shouted at & .802 & -.108 & -.175 & -.081 & .067 & -.125 & -.100 & .043 \\
\hline Talked privately & -.112 & .144 & .115 & .036 & .926 & .041 & .081 & .094 \\
\hline Confidentiality of information & -.040 & .155 & .145 & -.051 & .933 & .061 & .056 & -.020 \\
\hline Obtain information of friends/neighbors & .261 & .399 & -.109 & .554 & -.067 & -.207 & -.002 & .054 \\
\hline Confidentiality of records & .706 & .361 & -.175 & -.056 & .060 & -.209 & .029 & .070 \\
\hline Choice of provider & .073 & .259 & .122 & -.054 & -.082 & .626 & .073 & -.126 \\
\hline Choice of clinic & -.218 & -.543 & .370 & -.195 & .157 & .539 & .145 & .181 \\
\hline Able to go to other provider & -.216 & -.558 & .341 & -.191 & .167 & .546 & .149 & .147 \\
\hline Able to change provider & .686 & -.036 & .030 & .283 & .073 & .100 & .001 & -.335 \\
\hline Adequate information given & -.136 & .134 & .310 & -.124 & .110 & .060 & .846 & -.005 \\
\hline Freedom of choice & -.070 & .141 & .320 & -.018 & .099 & .032 & .870 & -.026 \\
\hline Second opinion & -.053 & .279 & -.011 & .045 & .121 & .606 & -.030 & .190 \\
\hline Discuss changing method & -.123 & .217 & -.088 & .871 & .079 & .053 & .129 & .123 \\
\hline Emergency FP & .024 & .010 & .048 & .946 & .056 & -.070 & -.045 & -.005 \\
\hline Discuss with spouse & .054 & .098 & .348 & .024 & .013 & .142 & -.061 & .655 \\
\hline Spouse can attend clinic & -.227 & .215 & .866 & .057 & .079 & .030 & .149 & .107 \\
\hline Family able to discuss & .639 & -.091 & -.107 & .221 & -.064 & .082 & .248 & -.075 \\
\hline Family able to obtain information & .748 & -.354 & .338 & -.219 & .105 & .259 & .016 & -.003 \\
\hline Spouse has time for questioning & .685 & -.123 & -.151 & .191 & -.109 & -.043 & -.088 & .055 \\
\hline
\end{tabular}

\section{Assessment of construct validity}

Response rate of the cross sectional survey was $100 \%$. In the analysis, 11 out of 28 items showed skewness and kurtosis, and robust maximum likelihood estimation was used for CFA. Linearity, muticolinearity and outliers were assessed for and the data was confirmed to be fit for factor analysis. ${ }^{3}$

Twelve models were assessed for goodness of fit as detailed in Table 2. All models did not fit the data well $(\mathrm{p}<0.000)$, but goodness of fit (GFI), non-normed fit index (NNFI) and comparative fit index (CFI) showed much improvement and standardized root mean square residual (SRMR) showed acceptable levels for model VII. Hence, model VII was accepted as the best fitting model (Fig. 1). The reliability was assessed measuring the internal consistency for the six domains and the details are shown in Table 3.

\section{Discussion}

Health system responsiveness is a novel and important concept in assessing the performance of health systems and a validated instrument was unavailable. In this study, both the quantitative and qualitative research methods provided comprehensive methodologies for exploration of ideas. Clients were the main focus of the development process. To discuss concepts with clients, FGDs were opted as it provided a suitable method to open discussion on a concept not widely known. ${ }^{4}$ The research was expanded to in-depth interviews since it provided opportunity to assess concept in-depth. ${ }^{4}$ Clients as well as other stakeholders improved the comprehensiveness of HESRAQ. A modified Delphi method provided a suitable methodology to finalize domains with views of a heterogeneous group. 
Table 2: Model fit statistics

\begin{tabular}{|lccccccc|}
\hline Model & & \multicolumn{7}{c}{ Fit Indices } \\
& $\chi 2$ & Df & P & GFI & NNFI & CFI & SRMR \\
I & 3056.86 & 351 & 0 & 0.48 & 0.55 & 0.58 & 0.17 \\
II & 2947.34 & 349 & 0 & 0.49 & 0.58 & 0.61 & 0.15 \\
III & 2965.35 & 349 & 0 & 0.48 & 0.56 & 0.60 & 0.17 \\
IV & 2752.46 & 344 & 0 & 0.50 & 0.59 & 0.63 & 0.17 \\
V & 2501.77 & 340 & 0 & 0.52 & 0.62 & 0.66 & 0.16 \\
VI & 2218.74 & 340 & 0 & 0.59 & 0.73 & 0.76 & 0.12 \\
VII & $\mathbf{1 2 7 2 . 4 9}$ & $\mathbf{3 3 5}$ & $\mathbf{0}$ & $\mathbf{0 . 6 9}$ & $\mathbf{0 . 8 4}$ & $\mathbf{0 . 8 6}$ & $\mathbf{0 . 0 7 5}$ \\
VIII & 1827.78 & 331 & 0 & 0.66 & 0.81 & 0.83 & 0.11 \\
IX & 1431.96 & 335 & 0 & 0.61 & 0.81 & 0.83 & 0.17 \\
X & 1587.44 & 329 & 0 & 0.69 & 0.84 & 0.86 & 0.074 \\
XI & 216.58 & 303 & 1.0 & - & - & 0.00 & - \\
XII & 10554 & 322 & 0 & -0.11 & 0.63 & 0.69 & 0.24 \\
\hline
\end{tabular}

$\chi 2=$ Satorra-Bentler scaled Chi-square test

$\mathrm{df}=$ degrees of freedom

$\mathrm{p}=\mathrm{p}$ value

$\mathrm{GFI}=$ goodness of fit index $(>0.9$ desired $)$

$\mathrm{NNFI}=$ Non-normed fit index $(>0.9$ desired $)$

$\mathrm{CFI}=$ comparative fit index $(>0.9$ desired $)$

$\mathrm{SRMR}=$ standardized root mean square residual $(<0.08$ desired $)$

Figure 1: Model VII
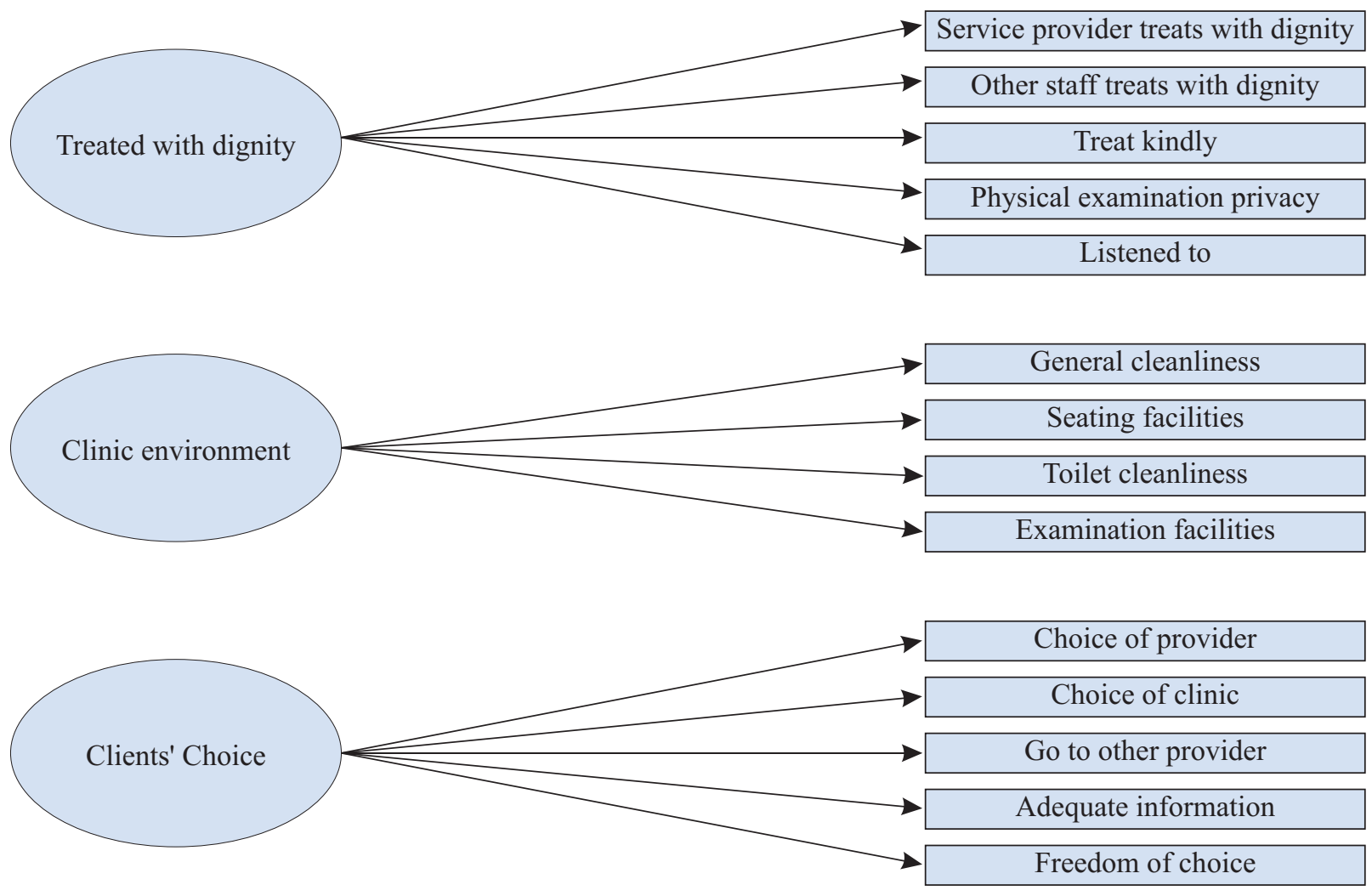

South East Asia Journal of Public Health 2011:1:46-52. 

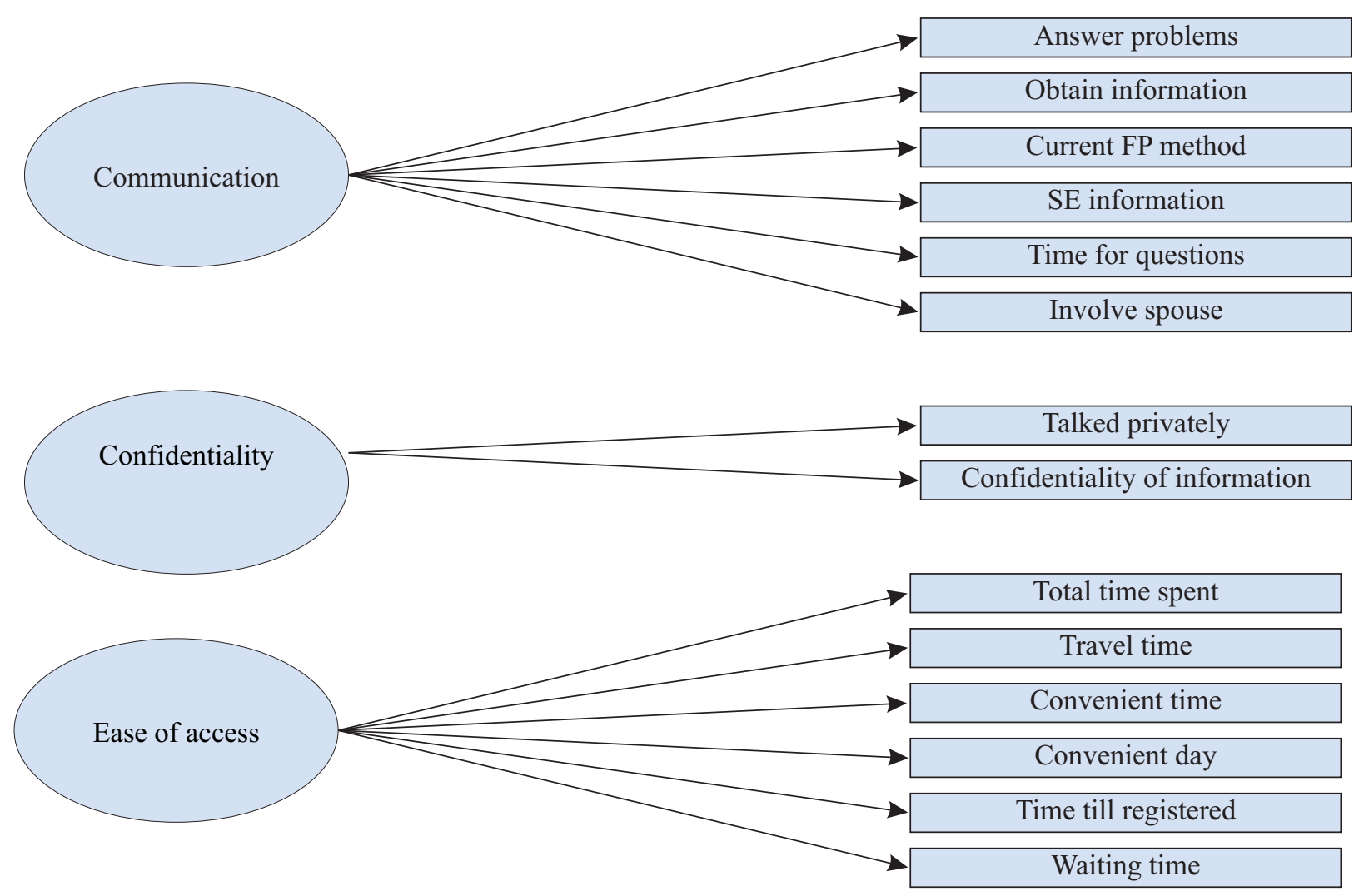

An exploration process revealed 15 domains and renaming five of the eight original domains improved suitability of the concept in the local setting. In the finalization, the use of a median score was justified due to the skewed nature of results. ${ }^{5}$ Though response rates were low in the Delphi process, views of at least one participant from each group of stakeholders was obtained. The finalized domains of the HSR were 'ease of access', 'treated with dignity', 'clinic environment', 'confidentiality', 'choice of the method', 'choice of the provider', 'communication' and 'family involvement'.

Table 3: Cronbach's alpha coefficients of the domains

\begin{tabular}{|lc|}
\hline Domain & Cronbach's alpha \\
Ease of access & 0.971 \\
Treated with dignity & 0.967 \\
Clients' choice & 0.951 \\
Communication & 0.877 \\
Confidentiality & 0.881 \\
Clinic environment & 0.951 \\
\hline
\end{tabular}

In developing a new tool, the items list should comprehensively cover different domains of the concept under assessment generating at least twice the number of items in the final instrument. The present study generated
92 items which can be considered as adequate. Items were reduced with opinion of experts and EFA with views of clients. Factor loadings above 0.5 were taken as the cutoff as items showed high correlations. Factors were identified based on the pattern of loading of items. The 28 items which loaded logically, indicating clients were able to relate items to the concept of the original domain, were retained for the instrument. The final list of items was reassessed by the panel of experts to assure domains were adequately represented and measured.

The concept of HSR does not have a meaningful gold standard. Therefore construct validity was used to validate HESRAQ. The factor structure of HSR was extensively explored and CFA was carried out on 12 models. Results proved model VII fitted the data better. This model was accepted as the best fitting model in the local context.

The six factor model retained four factors, namely 'ease of access', 'confidentiality', 'clinic environment' and 'being treated with dignity', with its original set of items. There was a combination of four factors. Factors, 'choice of provider' and 'choice of method' were combined indicating the clients understanding a common domain 'choice' as one nonmedical expectation. Domains 'communication' and 'family involvement' combined into a single factor. There was only one item, 'the ability to involve the spouse in discussions on FP with healthcare providers', in the domain 'family involvement' which had been understood as a component of communication. The WHO has also acknowledged that 
communication. The WHO has also acknowledged that there are many overlaps among domains though separated theoretically. ${ }^{1}$ Therefore the six factor model is acceptable in relation to family planning service provision in the local setting.

\section{Conclusion}

To examine health system responsiveness, a valid instrument is crucial. Using both qualitative and quantitative methodologies, the comprehensiveness of the HESRAQ was improved. The HESRAQ is a valid and reliable tool to assess the HSR of FP services in Sri Lanka and can be used the expectations of service seekers of other healthcare programs.

\section{References}

1. WHO. World Health Report 2000. Geneva: World Health Organization, 2000.

2. Redding CA, Maddock JE, Rossi JS. The sequential approach to measurement of health behavior constructs. Calif J Health Promot 2006; 4:83-101.

3. Staleton CD. Basic concepts and procedures of confirmatory factor analysis. Paper presented at Annual Meeting of the Southwest Educational Research Association. Austin, TX, 1997.

4. Britten, N. Qualitative research: qualitative interviews in medical research. $B M J 1995 ; 311: 251-3$.

5. Streiner DL, Norman GR. Health measurement scales: a practical guide to their development and use. London: Oxford University Press, 1995. 